\title{
Chronic Omega-3 Polyunsaturated Fatty Acid Treatment Variably Affects Cellular Repolarization in a Healed Post-MI Arrhythmia Model
}

\section{OPEN ACCESS \\ Edited by: \\ Sami Noujaim, \\ University of South Florida College of \\ Medicine, USA \\ Reviewed by: \\ Wayne Rodney Giles, \\ The University of Calgary, Canada \\ Elena Tolkacheva, \\ University of Minnesota, USA \\ Ruben Coronel, \\ Academic Medical Center, \\ Netherlands \\ ${ }^{*}$ Correspondence: \\ George E. Billman \\ billman.1@osu.edu; \\ Cynthia A. Carnes \\ carnes.4@osu.edu \\ ${ }^{\dagger}$ Present Address: \\ Yoshinori Nishijima, \\ Department of Medicine \\ Cardiovascular Center, Medical \\ College of Wisconsin, Milwaukee, WI, \\ USA \\ Pedro Vargas-Pinto,}

Universidad de la Salle, Bogotá,

Colombia

Specialty section:

This article was submitted to

Cardiac Electrophysiology,

a section of the journal

Frontiers in Physiology

Received: 19 October 2015 Accepted: 30 May 2016

Published: 14 June 2016

Citation:

Bonilla IM, Nishijima Y, Vargas-Pinto $P$, Baine SH, Sridhar A, Li C, Billman GE

and Carnes CA (2016) Chronic

Omega-3 Polyunsaturated Fatty Acid Treatment Variably Affects Cellular

Repolarization in a Healed Post-MI

Arrhythmia Model.

Front. Physiol. 7:225.

doi: 10.3389/fphys.2016.00225
Ingrid M. Bonilla ${ }^{1,2,3}$, Yoshinori Nishijima ${ }^{1 \dagger}$, Pedro Vargas-Pinto ${ }^{4 \dagger}$, Stephen H. Baine ${ }^{1}$, Arun Sridhar ${ }^{1}$, Chun $\mathrm{Li}^{5}$, George E. Billman ${ }^{2,3 *}$ and Cynthia A. Carnes ${ }^{1,2,3 *}$

${ }^{1}$ College of Pharmacy, The Ohio State University, Columbus, OH, USA, ${ }^{2}$ Department of Physiology and Cell Biology, The Ohio State University, Columbus, OH, USA, ${ }^{3}$ Dorothy M. Davis Heart and Lung Research Institute, The Ohio State University, Columbus, OH, USA, ${ }^{4}$ Department of Veterinary Biosciences, College of Veterinary Medicine, The Ohio State University, Columbus, OH, USA, ${ }^{5}$ Division of Cardiology, Peking University People's Hospital, Beijing, China

Introduction: Over the last 40 years omega-3 polyunsaturated fatty acids (PUFAs) have been shown to be anti-arrhythmic or pro-arrhythmic depending on the method and duration of administration and model studied. We previously reported that omega-3 PUFAs do not confer anti-arrhythmic properties and are pro-arrhythmic in canine model of sudden cardiac death (SCD). Here, we evaluated the effects of chronic omega-3 PUFA treatment in post-MI animals susceptible (VF+) or resistant (VF-) to ventricular tachyarrhythmias.

Methods: Perforated patch clamp techniques were used to measure cardiomyocyte action potential durations (APD) at 50 and $90 \%$ repolarization and short term variability of repolarization. The early repolarizing transient outward potassium current $I_{\text {to }}$ was also studied.

Results: Omega-3 PUFAs prolonged the action potential in VF- myocytes at both 50 and $90 \%$ repolarization. Short term variability of repolarization was increased in both untreated and treated VF- myocytes vs. controls. Ito was unaffected by omega-3 PUFA treatment. Omega-3 PUFA treatment attenuated the action potential prolongation in VF+ myocytes, but did not return repolarization to control values.

Conclusions: Omega-3 PUFAs do not confer anti-arrhythmic properties in the setting of healed myocardial infarction in a canine model of SCD. In canines previously resistant to ventricular fibrillation (VF-), omega-3 PUFA treatment prolonged the action potential in VF- myocytes, and may contribute to pro-arrhythmic responses.

Keywords: omega-3 polyunsaturated fatty acids, ventricular fibrillation, electrophysiology, potassium current, myocardial infarction

\section{INTRODUCTION}

Sudden cardiac death (SCD) caused by ventricular tachy-arrhythmias is the leading cause of death in the United States and contributes to $15-20 \%$ of deaths worldwide (Hayashi et al., 2015). Myocardial infarction and resulting scar tissue result in structural and electrical remodeling leading to an arrhythmogenic substrate; at the cellular level repolarization abnormalities and calcium 
dysregulation occur which can contribute to ventricular tachyarrhythmia formation and SCD (Rubart and Zipes, 2005; Wagner et al., 2015). Treatments that normalize repolarization may be anti-arrhythmic in the setting of healed MI and decrease the susceptibility to SCD (Billman, 2006).

The effects of omega-3 polyunsaturated fatty acid (PUFA) consumption and cardiovascular health have been studied for more than 40 years (Dyerberg et al., 1978). While observational studies suggest a role for omega-3 PUFAs in preventing cardiac mortality(Kromhout et al., 1985), results from studies using omega-3 PUFAs as secondary prevention of cardiovascular events have shown mixed results (Gillum et al., 1996; Erkkila et al., 2003; Wilhelm et al., 2008; Smith et al., 2009). Despite mixed results of prior studies the American Heart Association recommends eating fatty fish containing the active ingredients docosahexaenoic acid (DHA) and eicosapentaenoic acid (EPA) two times per week in patients with or without existing coronary heart disease (CHD) and $1 \mathrm{~g}$ of EPA +DHA by capsule per day in patients with existing CHD (Gebauer et al., 2006).

Cellular experiments have demonstrated that acute administration of omega-3 PUFAs are able to modulate a number ion currents including calcium, sodium, and potassium currents (Dhein et al., 2005; Guizy et al., 2005; Meves, 2008). However, it is not clear if these effects are pro-arrhythmic or anti-arrhythmic (Belevych et al., 2013). We previously reported that acute administration of omega-3 PUFAs through intravenous infusion of a fish oil emulsion prevented ventricular fibrillation in canines susceptible to induction of ventricular fibrillation (VF+) in a clinically relevant model of SCD (Leaf et al., 2003). In contrast, dietary administration of omega-3 PUFAs did not confer protection against VF (Billman et al., 2012). Furthermore, omega-3 PUFAs were shown to be proarrhythmic and cause SCD in the same animal model (Billman et al., 2012). Additionally, Coronel et al. reported that chronic dietary administration of omega-3 PUFAs were pro-arrhythmic and shortened the action potential in a regionally ischemic porcine model (Coronel et al., 2007).

The purpose of the current study was to elucidate the cellular electrophysiological effects caused by chronic dietary omega-3 PUFAs supplementation in a post-MI canine model of sudden death at both high $(\mathrm{VF}+)$ and low $(\mathrm{VF}-)$ risk of developing ventricular arrhythmias.

\section{MATERIALS AND METHODS}

All animal procedures were approved by The Ohio State University Institutional Animal Care and Use committee and conformed to the Guide for the Care and Use of Laboratory Animals published by the US National Institute of Health (NIH publication No. 85-23, revised 1996). Forty-three heartworm free mixed breed dogs had a left ventricular anterior myocardial infarction induced as previously described (Billman, 2006). Seventeen healthy age-matched dogs served as controls.

\section{In vivo Preparation}

The animal model, omega-3 PUFA treatment protocol, and in vivo assessments have been previously described (Sridhar et al.,
2008; Billman et al., 2012). Briefly, the left anterior descending coronary artery was occluded to induce a left anterior myocardial infarction (Billman et al., 1982; Billman, 2006). After recovery (34 weeks) the susceptibility to ventricular fibrillation was tested using a standardized exercise plus ischemia test (Billman, 2006; Babu et al., 2007; Sridhar et al., 2008). The exercise plus ischemia test reliably induced ventricular flutter/fibrillation in animals which were then classified as VF+. Those without induced sustained ventricular tachy-arrhythmias were classified as VF(Sridhar et al., 2008).

\section{Omega-3 PUFA Protocol}

To prevent confounding dietary omega-3 PUFA intake, dogs used for the omega-3 PUFA experiments were fed a diet free of omega-3 PUFAs (Harland Teklad, Harlan Laboratories, Inc., Indianapolis, IN, USA) for the duration of the study $(\sim 4$ months; Billman et al., 2010, 2012). Dogs were randomly assigned to the following groups: untreated $\mathrm{VF}+n=11$, untreated $\mathrm{VF}-n=8$, treated VF $+n=11$, and treated VF- $n=13$ (Figure 1). Dogs in the treated group received $465 \mathrm{mg}$ ethyl eicosapentaenoate, EPA + ethyl docosahexaenoate, DHA, $375 \mathrm{mg}$ per 1 g capsule (Lovaza ${ }^{\circledR}$, GlaxoSmithKline, Research Triangle

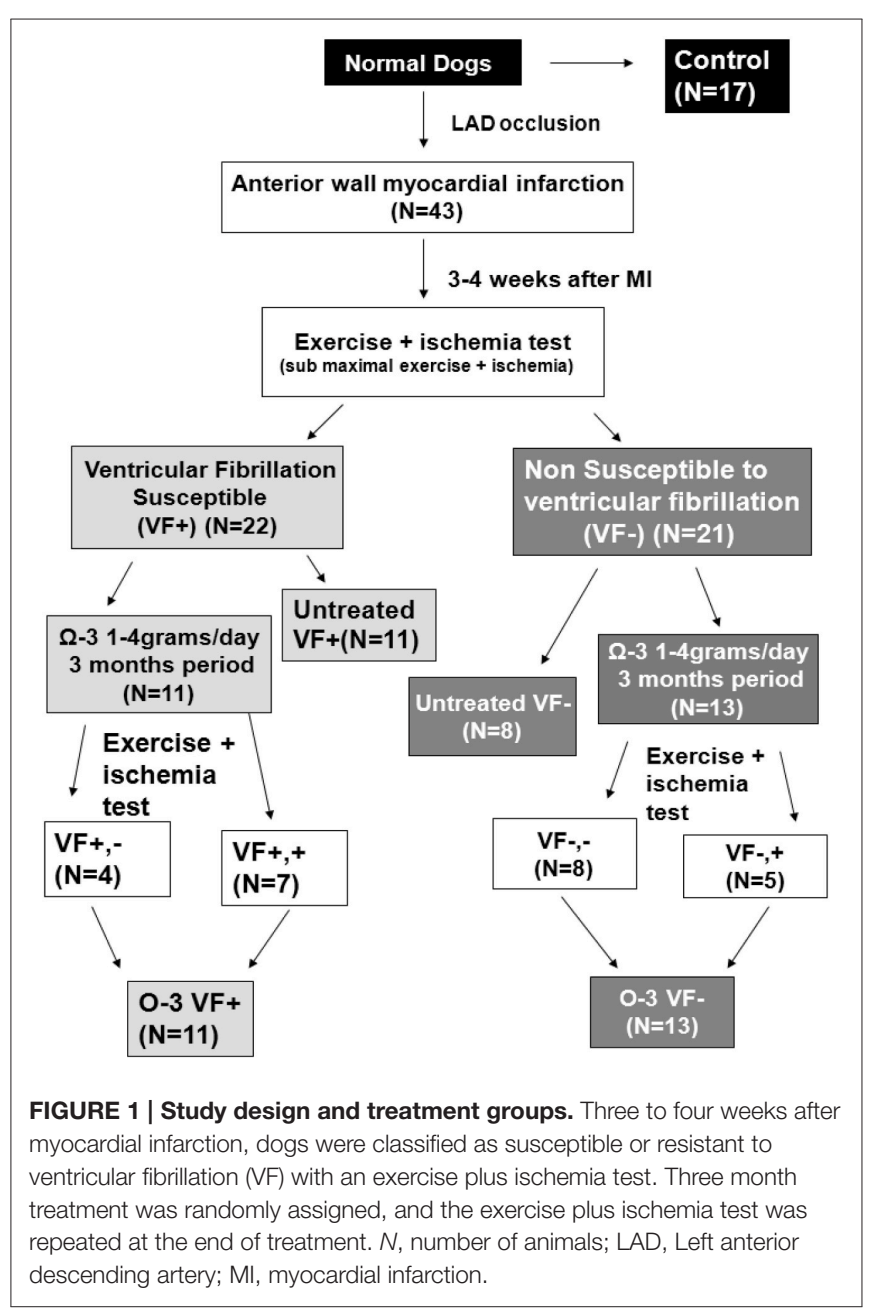


Park, NC); doses of 1,2 , and $4 \mathrm{~g}$ where administered. The capsules were given orally prior to the daily feeding (7 days per week for 3 months) as previously reported (Billman et al., 2012). The exercise plus ischemia test was repeated after the omega-3 PUFA treatment period (Billman et al., 2012). Since no dose-dependent differences in response were found in this or previous work in this model (Billman et al., 2010), results from all dose groups were combined. Omega-3 PUFA treatment induced malignant arrhythmias in $\sim 33 \%$ of the VF- dogs, indicating a pro-arrhythmic effect of treatment (Billman et al., 2012). In the VF+ dogs, omega-3 PUFA treatment did not confer any protection against malignant arrhythmias, as we previously reported (Billman et al., 2012). Since no significant cellular electrophysiological differences based on treatment response were found in either the VF- dogs or the VF+ dogs, canines were grouped as omega-3 PUFA treated VF- or VF+, respectively. Figure 1 provides a detailed schematic of the dog classification.

\section{Myocyte Isolation}

Myocytes were isolated as previously described (Sridhar et al., 2008; Bonilla et al., 2014). Briefly, left ventricular myocytes were isolated a minimum of 5 days after the final exercise plus ischemia test to avoid potential residual effects of acute ischemia. Dogs where anesthetized by intravenous injection of pentobarbital sodium (dosage: $120 \mathrm{mg} / \mathrm{kg}$ for the first $4.5 \mathrm{~kg}$ and $60 \mathrm{mg} / \mathrm{kg}$ for every 4.5 thereafter). After a deep plane of anesthesia was achieved, the heart was rapidly removed and perfused with cold cardioplegia solution containing the following in $\mathrm{mM}: \mathrm{NaCl} 110$, $\mathrm{CaCl}_{2} 1.2, \mathrm{KCl} 16, \mathrm{MgCl}_{2} 16$, and $\mathrm{NaHCO}_{3}$ 10. Cannulation of the left circumflex artery was used to perfuse the left ventricle as previously described (Bonilla et al., 2012). In post-MI dogs a clear margin of infarct was visible as scar tissue. After enzymatic digestion of the left ventricle (Bonilla et al., 2012; Belevych et al., 2013), the digested non-infarcted mid myocardial section (at least $1 \mathrm{~cm}$ from the scar border) was used as the source of myocytes. This method typically yielded $50-70 \%$ rod shaped ventricular myocytes. The myocytes were stored at room temperature in a standard incubation buffer containing the following in $\mathrm{mM}$ : $118 \mathrm{NaCl}, 4.8 \mathrm{KCl}, 1.2 \mathrm{MgCl}_{2}, 1.2 \mathrm{KH}_{2} \mathrm{PO}_{4}, 0.68$ glutamine, 10 glucose, 5 pyruvate, $1 \mathrm{CaCl}_{2}$, along with $1 \mu \mathrm{mol} / \mathrm{L}$ insulin, and $1 \%$ BSA until use. All myocyte electrophysiological experiments were conducted within $10 \mathrm{~h}$ of myocyte isolation.

\section{Electrophysiological Protocols}

To assess myocyte electrophysiology amphotericin-B perforated patch clamp techniques with a bath temperature of $36 \pm 0.5^{\circ} \mathrm{C}$ were used. Myocytes were placed in a laminin coated cell chamber (Cell Microcontrols, Norfolk, VA) and superfused with bath solution containing in mM: $135 \mathrm{NaCl}, 5 \mathrm{MgCl}_{2}, 5 \mathrm{KCl}, 10$ glucose, $1.8 \mathrm{CaCl}_{2}$, and 5 HEPES with $\mathrm{pH}$ adjusted to 7.40 with $\mathrm{NaOH}$. Borosilicate glass micropipettes with tip resistance of 1.5$3 \mathrm{M} \Omega$ were filled with pipette solution containing the following in mM: $100 \mathrm{~K}$-aspartate, $40 \mathrm{KCl}, 5 \mathrm{MgCl}, 5 \mathrm{EGTA}, 5 \mathrm{HEPES}, \mathrm{pH}$ adjusted to 7.2 with $\mathrm{KOH}$.

Action potential duration (APD) measurements were obtained from the average of the last 10 traces (steady state) from a train of 25 action potentials elicited at each stimulation rate. To evaluate beat to beat variability, Poincaré plots of the last 10 consecutive beats were drawn by plotting each APD90 (APD90 $n+1$ ) against the APD90 of the previous beat (APD90 $n$ ) as previously reported (Varkevisser et al., 2012; Belevych et al., 2013). Short term variability of APD90, expressed in $\mathrm{ms}$, was calculated by using the following formula: $\sum(A P D 90 n+1-A P D 90 n) /(10 \times \sqrt{2})$ as previously reported (Varkevisser et al., 2012; Belevych et al., 2013).

For $\mathrm{I}_{\text {to }}$ recordings, only recordings with an access resistance $<20 \mathrm{M} \Omega$ were included in the analyses. Calcium in the bath solution was reduced to $1.0 \mathrm{mM}$ and $2 \mu \mathrm{M}$ nifidepine was added to block L-type $\mathrm{Ca}^{2+}$ current. $\mathrm{I}_{\text {to }}$ was elicited from a holding potential of $-60 \mathrm{mV}$ by a series of $100 \mathrm{~ms}$ test potentials from -30 to $+50 \mathrm{mV}$ and measured as peak current minus steady state current as previously described (Belevych et al., 2013).

\section{Solutions and Chemicals}

All chemicals used for buffer and solution preparation were purchased from Sigma Aldrich (St. Louis, CS) unless otherwise noted. Cardioplegia and solutions containing nifidepine and amphotericin-B were prepared daily.

\section{Interstitial Fibrosis}

Transmural right ventricular tissue samples were formalin fixed and embedded in paraffin and sectioned to $5 \mu \mathrm{m}$ thickness, using standard procedures. Tissue sections were stained with Masson's Trichrome to define the percentage area of fibrosis, as previously described (Nishijima et al., 2007).

\section{Data Analysis}

Cellular electrophysiology data were analyzed using Clampfit 10.3 software (Axon Instruments) and Origin 9.0 software (OriginLab, Northampton, MA, USA). Comparisons between three or more groups were analyzed by one-way ANOVA with post hoc least significant difference testing (Originpro 8.6, OriginLab). All data are presented as mean $\pm \mathrm{SE}$ and $p<0.05$ was the criterion for statistical significance for all comparisons.

\section{RESULTS}

\section{Omega-3 PUFA Treatment of VF-Canines Causes a Prolongation of Cellular Repolarization}

APD at $90 \%$ repolarization (APD90) in untreated VF- cells was significantly prolonged compared to control at 0.5 and $2 \mathrm{~Hz}(p<$ $0.05)$; no differences were observed in APD at $50 \%$ repolarization (APD50; Figure 2). However, omega-3 PUFA treatment of VFdogs caused a significant prolongation of APD50 at 0.5 and $1 \mathrm{~Hz}$ $(p<0.05$ vs. control and untreated VF- $)$ and increased APD90 at all rates compared to untreated VF- $(p<0.05$ vs. untreated $\mathrm{VF}-$ and control; Figure 2 ). Short term variability was measured to assess the instability of the action potential, a measure of pro-arrhythmic potential. Both untreated VF- and the omega3 PUFA treated VF- groups had increased short term variability vs. control $(p<0.05)$, while omega-3 PUFA treatment did not affect short term variability (Figure 2 ). 
A

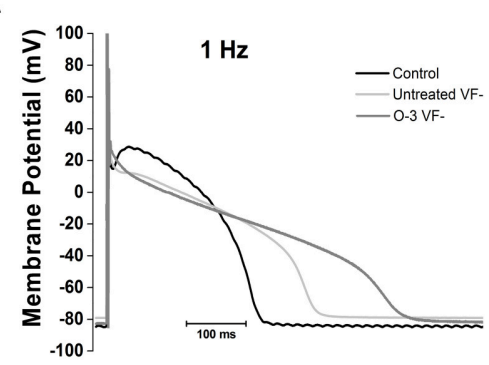

C

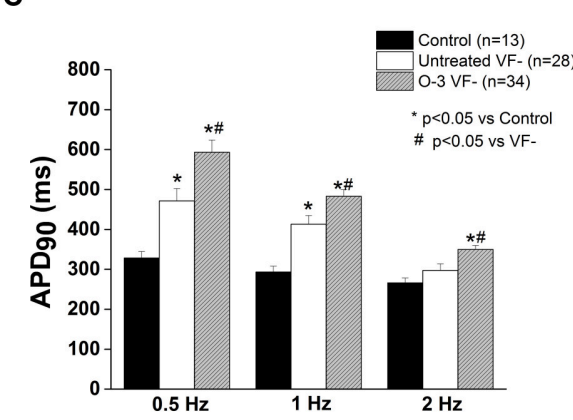

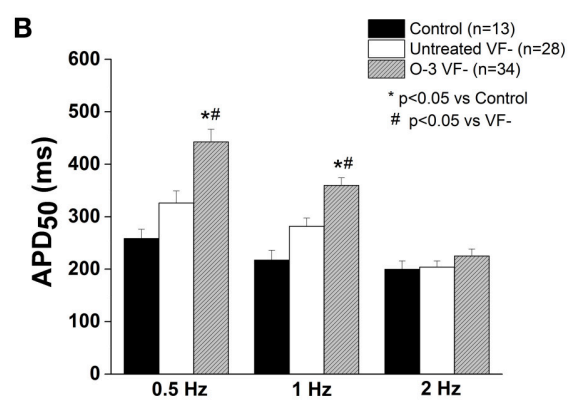

D

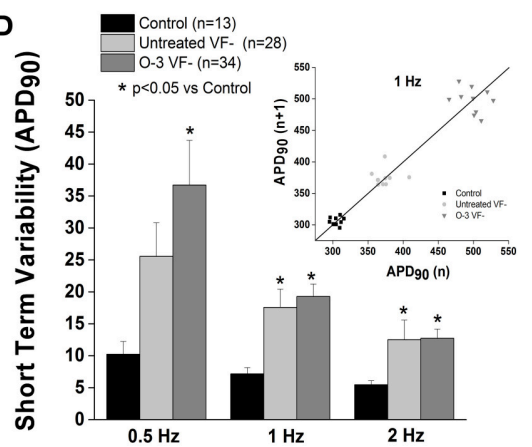

FIGURE 2 | Omega-3 PUFA treatment prolongs repolarization in VF- myocytes. (A) Representative tracings of action potentials of control, untreated VF-, and omega-3 PUFA treated VF- cardiomyocytes. (B) APD 50 is significantly increased in the omega-3 PUFA treated VF- myocytes compared to both control and untreated VF- cardiomyocytes $(P<0.05$ vs. control and untreated VF- 0.5 and $1 \mathrm{~Hz}$ ). (C) APDgo is significantly increased in the omega-3 PUFA treated group at all rates; compared to both control and untreated VF- cardiomyocytes $\left(P<0.05\right.$ vs. control and untreated VF- at $0.5,1$, and $2 \mathrm{~Hz}$ ). (D) Short term variability of $A P D_{90}$ is not further increased by omega-3 PUFA treatment $(P<0.05$ vs. control at all rates). Insert: Representative Poincaré Plot of APD 90 from control, Untreated VF- and O-3 VF- groups, $6-8$ animals per group, $n=$ number of cells.

\section{Cardiac Transient Outward Potassium Current $\left(I_{\text {to }}\right)$ Is Unaffected by Omega-3 PUFA Treatment in VF- Animals}

In accordance with our previous study, $I_{\text {to }}$ current was significantly reduced in the untreated VF- group compared to control ( $p<0.05$ vs. control). Omega-3 PUFA treatment had no effect on $\mathrm{I}_{\text {to }}$ current vs. untreated VF- canines (Figure 3).

\section{Omega-3 PUFA Treatment of Animals Susceptible to Ventricular Arrhythmias (VF+) Causes a Shortening of the Action Potential}

Previously we found that myocytes from animals susceptible to ventricular fibrillation ( $\mathrm{VF}+$ ) had a significantly prolonged APD50 and APD90 compared to controls (Sridhar et al., 2008). Omega-3 PUFA treatment caused a significant shortening of the APD50 $(1 \mathrm{~Hz})$ and APD90 $(0.5$ and $1 \mathrm{~Hz})$ compared to untreated $\mathrm{VF}+$, however, repolarization remained prolonged vs. control $(p<0.05$ vs. control and untreated VF + ). At $2 \mathrm{~Hz}$ omega-3 PUFA treatment did not alter APD (Figure 4).

Short term variability of the APD at $90 \%$ of repolarization (STV) in VF+ myocytes was significantly increased compared to control myocytes at 1 and $2 \mathrm{~Hz}(P<0.05$ vs. control). Omega- 3 PUFA treatment caused a significant reduction of STV at $1 \mathrm{~Hz}$, yet was increased relative to controls ( $p<0.05$ vs. control and untreated VF+, Figure 4).

\section{Cardiac Transient Outward Potassium Current (Ito) Is Unaffected by Omega-3 PUFA Treatment in VF+ Animals}

Consistent with our previous report (Sridhar et al., 2008), I Io was reduced in myocytes from VF+ canines ( $p<0.5$ vs. control; Figure 5). Omega-3 PUFA treatment had no effect on $I_{\text {to }}$ vs. untreated $\mathrm{VF}+$ canines.

\section{Interstitial Fibrosis}

Right ventricular tissue fibrosis was $14.0 \pm 1.1 \%$, and unchanged by infarction in either the VF- group $(10.5 \pm 1.2 \%)$ or VF+ group $(10.8 \pm 5.6 \%)$. Omega-3 PUFA treatment did not alter fibrosis in either the VF- or VF+ group (data not shown, $p=\mathrm{NS})$.

\section{DISCUSSION}

In the present study we tested the hypothesis that dietary omega3 PUFA supplementation would mediate electrophysiologic effects evident at the cellular level in the post-infarction setting. A major finding of this study is that in post-MI animals resistant to VF, omega-3 PUFA treatment caused a significant prolongation of the action potential and did not have beneficial effects 

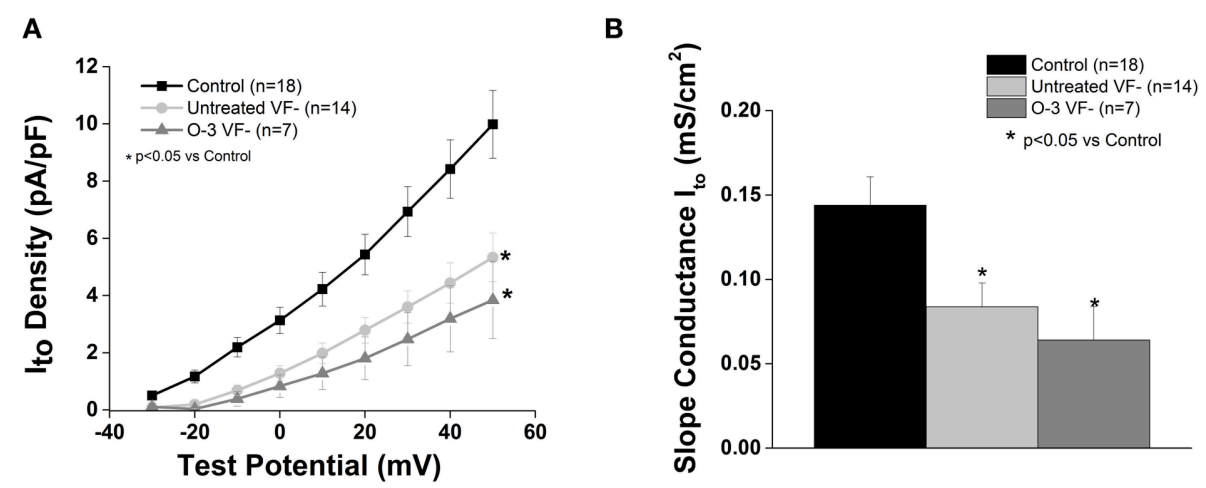

FIGURE 3 | Omega-3 PUFA treatment does not affect $\mathbf{I}_{\text {to }}$ in myocytes from VF- animals. (A) I-V curve. (B) Slope conductance. Ito density and slope conductance is significantly lower in VF- vs. control cardiomyocytes and unaffected by omega-3 PUFA treatment ( $P<0.05$ vs. control). Three to six animals per group; $n=$ number of cells.

A

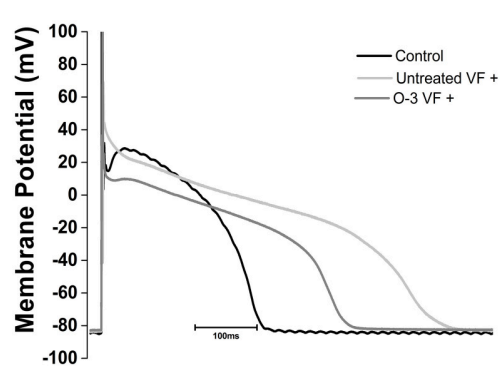

C

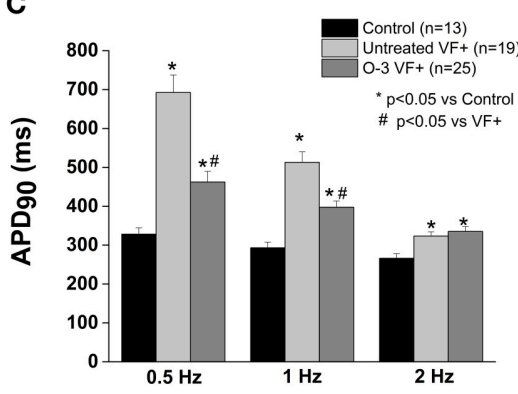

B

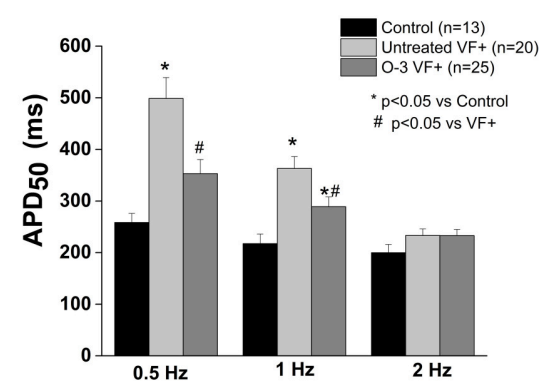

D

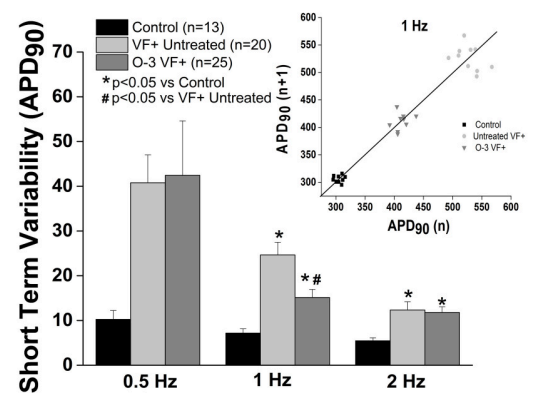

FIGURE 4 | Omega-3 PUFA treatment attenuates action potential duration prolongation in myocytes from VF+ myocytes. (A) Representative tracings of action potentials of control, untreated $\mathrm{VF}+$ and omega-3 PUFA treated $\mathrm{VF}+$ cardiomyocytes. (B) $\mathrm{APD}_{50}$ is prolonged in $\mathrm{VF}+$ myocytes at 0.5 and $1 \mathrm{~Hz}$, the prolongation is attenuated only at $1 \mathrm{~Hz}(P>0.05)$. (C) APD 90 is significantly prolonged in the VF+ group; omega-3 PUFA treatment attenuated the prolongation at 0.5 and $1 \mathrm{~Hz}(P<0.05)$. (D) Short term variability of APDgo is decreased only at $1 \mathrm{~Hz}$, but remains significantly higher than in control cardiomyocytes $(P<0.05 \mathrm{vs}$. control at $1 \mathrm{~Hz}$ ). Insert shows a representative Poincaré Plot of APDgo at $1 \mathrm{~Hz}$ from control, Untreated VF+ and O-3 VF+ groups. Five to seven animals per group; $n=$

number of cells.

on repolarization instability, consistent with pro-arrhythmic potential. Additionally, dietary Omega-3 PUFAs significantly shortened the action potential of dogs susceptible to VF (VF+) compared to untreated dogs, but did not consistently improve repolarization instability. Collectively, while we did observe cellular electrophysiologic effects of omega-3 PUFA treatment, these were insufficient to protect against malignant arrhythmias induced by acute myocardial ischemia in vivo in the VF+ animals.
Epidemiological studies from Bang and Dyerberg suggests beneficial results from the intake of omega-3 PUFAs, based on initial observations in a population of Greenland Inuits (Bang et al., 1971). This led to the hypothesis that increased omega-3 PUFA consumption is inversely correlated with SCD, although more recent data suggests genetic adaptations to dietary omega3 PUFAs may exist in this population, and may contribute to the observed effects (Fumagalli et al., 2015). Early clinical and investigational trials confirmed this inverse relationship. For 

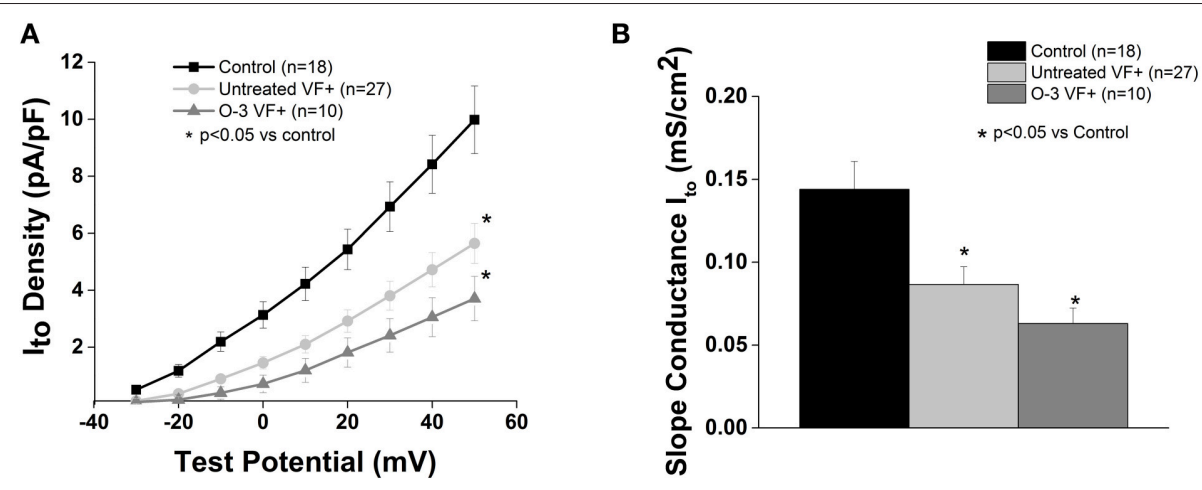

FIGURE 5 | Omega-3 PUFA treatment does not affect $\mathbf{I}_{\text {to }}$ in VF+ myocytes. (A) I-V curve and (B) Slope conductance of control, untreated VF+, and omega-3 PUFA treated VF+ cardiomyocytes. I $_{\text {to }}$ is reduced in VF+ myocytes and unaffected by omega-3 PUFA treatment $(P<0.05$ vs. control). Three to seven animals per group; $n=$ number of cells.

example, the GISSI-Prevenzione trial reported a $45 \%$ reduction in sudden death in post-MI patients treated with omega-3 PUFA supplements (Marchioli et al., 2002). However, recent clinical trials for the secondary prevention of adverse cardiovascular events following myocardial infarction such as the OMEGA and Alpha-Omega trial have not replicated the earlier success seen in the GISSI-Prevenzione study (Billman, 2013). In addition, animal studies have not provided a clear consensus on the pro- or anti-arrhythmic properties of omega-3 PUFAs (Billman, 2013). This may be attributed to differences in acute vs. chronic administration of omega-3 PUFAs (Billman, 2013). While studies have shown that acute application of omega-3 PUFAs reduce several cardiac ionic currents including: $\mathrm{I}_{\mathrm{to}}, \mathrm{I}_{\mathrm{Na}}$, and $\mathrm{I}_{\mathrm{Kr}}$, the generalizability of these results to chronic supplementation is not clear (Xiao et al., 1995; Jude et al., 2003; Guizy et al., 2005). For example, a recent report shows that acute application of omega3 PUFAs in COS7 cells increases Kv.7.1/KCNE1 (subunit which encodes slow delayed rectifying potassium channel $\mathrm{I}_{\mathrm{Ks}}$ ) while chronic application modified channel activity resulting in proarrhythmic behavior (Moreno et al., 2015). In the present study, and in the companion in vivo studies (Billman et al., 2010, 2012) we sought to evaluate the role of chronic supplementation, and the consequent membrane incorporation of omega-3 PUFAs, in a canine model of SCD.

It is known that action potential prolongation in certain settings may favor the development of triggered arrhythmias (Rubart and Zipes, 2005). However, repolarization changes may be pro- or anti-arrhythmic depending on the underlying substrate (den Ruijter et al., 2007). Abnormal repolarization may occur in individual cells or at the tissue level with resulting increases in the propensity for SCD, which uncoupled cells being more susceptible to arrhythmias (Rubart and Zipes, 2005). In VF- animals, chronic omega-3 PUFA treatment resulted in pro-arrhythmic actions, both in vivo (Billman et al., 2012) and at the isolated myocyte level as shown in the present publication. An interesting finding of the present study is the discrepancy between modulation of APD and arrhythmic potential. Although, the VF+ myocytes did show attenuation of AP prolongation after omega-3 PUFA treatment, this was insufficient to translate to a reduced arrhythmia potential. It is possible that the magnitude of attenuated AP prolongation was insufficient to protect against malignant arrhythmias induced by acute myocardial ischemia in vivo. We used short term variability or beat to beat variability of APD as an ex vivo parameter to measure the instability of repolarization (Oosterhoff et al., 2007). Increases in short term variability of repolarization has shown to be predictive of ventricular arrhythmias in canine and human models of disease, when repolarization is compromised and early after depolarizations contribute to arrhythmogenesisas we have previously reported occurs in the VF+ model (Oosterhoff et al., 2007; Sridhar et al., 2008). Our data suggest that increased myocyte repolarization variability may correspond to the previously observed pro-arrhythmic risk.

We have previously reported that chronic omega-3 PUFA treatment in this model results in dysregulation of intracellular calcium handling (Belevych et al., 2013), a potential contributor to cellular arrhythmias. Collectively this suggests that at the cellular level calcium dysregulation, rather than abnormal repolarization in isolation, may contribute to the lack of anti-arrhythmic efficacy of this treatment approach, while contributing to pro-arrhythmic potential in some subjects.

Cardiac transient outward potassium current $\mathrm{I}_{\text {to }}$ is responsible for partial repolarization during phase 1 of the cardiac action potential (Niwa and Nerbonne, 2010). We previously reported that a reduction in $\mathrm{I}_{\text {to }}$ alone was seen in VF- myocytes, and therefore is insufficient to increase arrhythmia susceptibility in this model (Sridhar et al., 2008). However, block of an additional repolarizing current $\left(\mathrm{I}_{\mathrm{Kr}}\right)$ was sufficient to induce cellular arrhythmias in VF- myocytes (Sridhar et al., 2008). This suggests a role for abnormal "repolarization reserve" as part of the substrate for arrhythmias in this post-MI model. In the present study we evaluated $I_{\text {to }}$ with dietary omega-3 PUFA supplementation in a canine model of sudden death, and there was no effect on $\mathrm{I}_{\text {to }}$ in either group. This finding is consistent with our previous results (Sridhar et al., 2008) and the notion that reduced $\mathrm{I}_{\text {to }}$ does not play a significant role in altering lability of repolarization in canine models unless other electrophysiologic parameters are altered. It is therefore likely that other repolarizing currents are augmented by omega3 PUFA treatment resulting in shortened APD in the VF+ 
myocytes. Verkerk et al. showed that chronic omega-3 PUFA treatment in male pigs resulted in increased outward $\mathrm{I}_{\mathrm{K} 1}$ and $\mathrm{I}_{\mathrm{Ks}}$, both of which would contribute to accelerated repolarization; these currents may contribute to the accelerated repolarization we observed in the VF+ omega-3 PUFA treated group (Verkerk et al., 2006). Omega-3 PUFAs have been suggested to have possible anti-fibrotic effects (Wang and O'Horo, 2011; Eclov et al., 2015). Our data do not support an anti-fibrotic effect of omega-3 PUFA treatment, although our post-MI model does not result in significant right ventricular fibrosis and may limit the ability to detect an anti-fibrotic response.

\section{LIMITATIONS OF THE STUDY}

The American Heart Association recommends $1 \mathrm{~g}$ of EPA+ DHA supplementation for patients with existing CHD. Identifying dose equivalents between animals and humans remains a challenge (Billman, 2013). The 1-4 g EPA + DHA given to canines in the present study would be considered higher than the normal human equivalent for individuals with existing CHD. However, dosage used in the present study reflects previously reported RBC and cardiac tissue levels (Billman, 2012) that are associated with a reduction in sudden death in epidemiological studies (Siscovick et al., 1995; Albert et al., 2002).

This study did not comprehensively evaluate ion currents contributing to APD. Rather the integrated response to ion currents, the action potential was used to evaluate the response to chronic omega-3 PUFA treatment, within a rate range of 30120 BPM. While these rates capture the majority of the daily heart rate range in canines, it is possible that results at higher rates, which may occur physiologically in dogs, would have different results. Isolated myocytes are by definition uncoupled and therefore may have intrinsically longer repolarization and more repolarization instability. Tissue recordings in coupled myocardium would be required to more closely reflect the cellular repolarization in vivo. Another consideration for interpreting the in vivo effects of omega-3 PUFAs is the potential for modulation of connexin activity (Baum et al., 2012; Radosinska et al., 2013), a possibility not examined in our model.

\section{REFERENCES}

Albert, C. M., Campos, H., Stampfer, M. J., Ridker, P. M., Manson, J. E., Willett, W. C., et al. (2002). Blood levels of long-chain n-3 fatty acids and the risk of sudden death. N. Engl. J. Med. 15, 1113-1118. doi: 10.1056/NEJMoa012918

Babu, G. J., Bhupathy, P., Carnes, C. A., Billman, G. E., and Periasamy, M. (2007). Differential expression of sarcolipin protein during muscle development and cardiac pathophysiology. J. Mol. Cell Cardiol. 2, 215-222. doi: 10.1016/j.yjmcc.2007.05.009

Bang, H. O., Dyerberg, J., and Nielsen, A. B. (1971). Plasma lipid and lipoprotein pattern in Greenlandic West-coast Eskimos. Lancet 7710, 1143-1145. doi: 10.1016/S0140-6736(71)91658-8

Baum, J. R., Dolmatova, E., Tan, A., and Duffy, H. S. (2012). Omega 3 fatty acid inhibition of inflammatory cytokine-mediated connexin 43 regulation in the heart. Front. Physiol. 3:272. doi: 10.3389/fphys.2012.00272

Belevych, A. E., Ho, H. T., Terentyeva, R., Bonilla, I. M., Terentyev, D., Carnes, C. A., et al. (2013). Dietary omega-3 fatty acids promote arrhythmogenic remodeling of cellular $\mathrm{Ca}^{2+}$ handling in a postinfarction model of

\section{CONCLUSION}

Chronic administration of omega-3 PUFAs results in variable electrophysiologic responses. Our previous in vivo reports in this canine model show that such treatments may be ineffective in VF+ animals or pro-arrhythmic in VF- animals (Sridhar et al., 2008; Billman, 2012; Billman et al., 2012). We previously reported that incorporated omega-3 PUFAs after chronic treatment increased the risk of developing arrhythmias by disrupting myocyte calcium handling (Belevych et al., 2013). Therefore, abnormal variations in calcium regulation in $\mathrm{VF}-$ myocytes could contribute to the pro-arrhythmic effects of dietary omega3 PUFAs in a canine model of sudden death. Abnormal calcium handling in tandem with altered repolarization may contribute to the observed effects of omega-3 PUFA supplementation. Our work does not support the routine use of chronic omega-3 PUFA supplements post-MI to reduce the risk of ventricular tachy-arrhythmias.

\section{AUTHOR CONTRIBUTIONS}

IB: data collection and analysis; wrote portions of the paper; final approval of manuscript. YN, PV, and AS: data collection and analysis; critical revision of manuscript; final approval of manuscript. SB: data analysis, wrote, and revised sections of the manuscript; final approval of manuscript. CL: data collection and analysis; final approval of manuscript. GB: designed experimental protocol, oversaw all data collection and analysis, participated in drafting manuscript; approved final version of manuscript. CC: designed experimental protocol, conducted in vivo studies, participated in drafting, and approved preparation of final version of manuscript.

\section{ACKNOWLEDGMENTS}

This study was supported in part from National Institutes of Health by grants HL089836 (to CC), and HL086700 (to GB). The authors would like to thank Jeanne Green, RVT for technical assistance.

sudden cardiac death. PLoS ONE 10:e78414. doi: 10.1371/journal.pone. 0078414

Billman, G. E. (2006). A comprehensive review and analysis of 25 years of data from an in vivo canine model of sudden cardiac death: implications for future anti-arrhythmic drug development. Pharmacol. Ther. 3, 808-835. doi: 10.1016/j.pharmthera.2006.01.002

Billman, G. E. (2012). Effect of dietary omega-3 polyunsaturated fatty acids on heart rate and heart rate variability in animals susceptible or resistant to ventricular fibrillation. Front. Physiol. 3:71. doi: 10.3389/fphys.2012.00071

Billman, G. E. (2013). The effects of omega-3 polyunsaturated fatty acids on cardiac rhythm: a critical reassessment. Pharmacol. Ther. 1, 53-80. doi: 10.1016/j.pharmthera.2013.05.011

Billman, G. E., Carnes, C. A., Adamson, P. B., Vanoli, E., and Schwartz, P. J. (2012). Dietary omega-3 fatty acids and susceptibility to ventricular fibrillation: lack of protection and a proarrhythmic effect. Circ. Arrhythm. Electrophysiol. 3, 553-560. doi: 10.1161/CIRCEP.111.966739

Billman, G. E., Nishijima, Y., Belevych, A. E., Terentyev, D., Xu, Y., Haizlip, K. M., et al. (2010). Effects of dietary omega-3 fatty acids on ventricular function 
in dogs with healed myocardial infarctions: in vivo and in vitro studies. Am. J. Physiol. Heart Circ. Physiol. 4, H1219-H1228. doi: 10.1152/ajpheart.01065.2009

Billman, G. E., Schwartz, P. J., and Stone, H. L. (1982). Baroreceptor reflex control of heart rate: a predictor of sudden cardiac death. Circulation 4, 874-880. doi: 10.1161/01.CIR.66.4.874

Bonilla, I. M., Belevych, A. E., Sridhar, A., Nishijima, Y., Ho, H. T., He, Q., et al. (2012). Endurance exercise training normalizes repolarization and calcium-handling abnormalities, preventing ventricular fibrillation in a model of sudden cardiac death. J. Appl. Physiol (1985). 11, 1772-1783. doi: 10.1152/japplphysiol.00175.2012

Bonilla, I. M., Vargas-Pinto, P., Nishijima, Y., Pedraza-Toscano, A., Ho, H. T., Long, V. P. III, et al. (2014). Ibandronate and ventricular arrhythmia risk. J. Cardiovasc. Electrophysiol. 3, 299-306. doi: 10.1111/jce.12327

Coronel, R., Wilms-Schopman, F. J., den Ruijter, H. M., Belterman, C. N., Schumacher, C. A., Opthof, T., et al. (2007). Dietary n-3 fatty acids promote arrhythmias during acute regional myocardial ischemia in isolated pig hearts. Cardiovasc. Res. 2, 386-394. doi: 10.1016/j.cardiores.2006.10.006

den Ruijter, H. M., Berecki, G., Opthof, T., Verkerk, A. O., Zock, P. L., and Coronel, R. (2007). Pro- and antiarrhythmic properties of a diet rich in fish oil. Cardiovasc. Res. 2, 316-325. doi: 10.1016/j.cardiores.2006.06.014

Dhein, S., Michaelis, B., and Mohr, F. W. (2005). Antiarrhythmic and electrophysiological effects of long-chain omega-3 polyunsaturated fatty acids. Naunyn Schmiedebergs Arch. Pharmacol. 3, 202-211. doi: 10.1007/s00210-0051024-z

Dyerberg, J., Bang, H. O., Stoffersen, E., Moncada, S., and Vane, J. R. (1978). Eicosapentaenoic acid and prevention of thrombosis and atherosclerosis? Lancet 8081, 117-119.

Eclov, J. A., Qian, Q., Redetzke, R., Chen, Q., Wu, S. C., Healy, C. L., et al. (2015). EPA, not DHA, prevents fibrosis in pressure overload-induced heart failure: potential role of free fatty acid receptor 4. J. Lipid Res. 12, 2297-2308. doi: 10.1194/jlr.M062034

Erkkila, A. T., Lehto, S., Pyorala, K., and Uusitupa, M. I. (2003). n-3 fatty acids and 5 -y risks of death and cardiovascular disease events in patients with coronary artery disease. Am. J. Clin. Nutr. 1, 65-71.

Fumagalli, M., Moltke, I., Grarup, N., Racimo, F., Bjerregaard, P., Jorgensen, M. E., et al. (2015). Greenlandic Inuit show genetic signatures of diet and climate adaptation. Science 6254, 1343-1347. doi: 10.1126/science.aab2319

Gebauer, S. K., Psota, T. L., Harris, W. S., and Kris-Etherton, P. M. (2006). n-3 fatty acid dietary recommendations and food sources to achieve essentiality and cardiovascular benefits. Am. J. Clin. Nutr. 83(Suppl. 6), 1526S-1535S.

Gillum, R. F., Mussolino, M. E., and Madans, J. H. (1996). The relationship between fish consumption and stroke incidence. The NHANES I Epidemiologic Followup Study (National Health and Nutrition Examination Survey). Arch. Intern. Med. 5, 537-542.

Guizy, M., Arias, C., David, M., Gonzalez, T., and Valenzuela, C. (2005). \{Omega\}-3 and \{omega\}-6 polyunsaturated fatty acids block HERG channels. Am. J. Physiol Cell Physiol. 5, C1251-C1260. doi: 10.1152/ajpcell.00036.2005

Hayashi, M., Shimizu, W., and Albert, C. M. (2015). The spectrum of epidemiology underlying sudden cardiac death. Circ. Res. 12, 1887-1906. doi: 10.1161/CIRCRESAHA.116.304521

Jude, S., Bedut, S., Roger, S., Pinault, M., Champeroux, P., White, E., et al. (2003). Peroxidation of docosahexaenoic acid is responsible for its effects on I TO and I SS in rat ventricular myocytes. Br. J. Pharmacol. 4, 816-822. doi: 10.1038/sj.bjp.0705308

Kromhout, D., Bosschieter, E. B., and de Lezenne, C. C. (1985). The inverse relation between fish consumption and 20-year mortality from coronary heart disease. N. Engl. J. Med. 19, 1205-1209.

Leaf, A., Kang, J. X., Xiao, Y. F., and Billman, G. E. (2003). Clinical prevention of sudden cardiac death by $n-3$ polyunsaturated fatty acids and mechanism of prevention of arrhythmias by n-3 fish oils. Circulation 21, 2646-2652. doi: 10.1161/01.CIR.0000069566.78305.33

Marchioli, R., Barzi, F., Bomba, E., Chieffo, C., Di, G. D., Di, M. R., et al. (2002). Early protection against sudden death by $n-3$ polyunsaturated fatty acids after myocardial infarction: time-course analysis of the results of the Gruppo Italiano per lo Studio della Sopravvivenza nell'Infarto Miocardico (GISSI)-Prevenzione. Circulation 16, 1897-1903. doi: 10.1161/01.CIR.0000014682.14181.F2

Meves, H. (2008). Arachidonic acid and ion channels: an update. Br. J. Pharmacol. 1, 4-16. doi: 10.1038/bjp.2008.216
Moreno, C., de la Cruz, A., Oliveras, A., Kharche, S. R., Guizy, M., Comes, N., et al. (2015). Marine n-3 PUFAs modulate IKs gating, channel expression, and location in membrane microdomains. Cardiovasc. Res. 2, 223-232. doi: 10.1093/cvr/cvu250

Nishijima, Y., Sridhar, A., Viatchenko-Karpinski, S., Shaw, C., Bonagura, J. D., Abraham, W. T., et al. (2007). Chronic cardiac resynchronization therapy and reverse ventricular remodeling in a model of nonischemic cardiomyopathy. Life Sci. 14, 1152-1159. doi: 10.1016/j.lfs.2007.08.022

Niwa, N., and Nerbonne, J. M. (2010). Molecular determinants of cardiac transient outward potassium current (I(to)) expression and regulation. J. Mol. Cell Cardiol. 1, 12-25. doi: 10.1016/j.yjmcc.2009.07.013

Oosterhoff, P., Oros, A., and Vos, M. A. (2007). Beat-to-beat variability of repolarization: a new parameter to determine arrhythmic risk of an individual or identify proarrhythmic drugs. Anadolu. Kardiyol. Derg. 73-78. doi: 10.1016/j.jacc.2006.05.048

Radosinska, J., Bacova, B., Knezl, V., Benova, T., Zurmanova, J., Soukup, T., et al. (2013). Dietary omega-3 fatty acids attenuate myocardial arrhythmogenic factors and propensity of the heart to lethal arrhythmias in a rodent model of human essential hypertension. J. Hypertens. 9, 1876-1885. doi: 10.1097/HJH.0b013e328362215d

Rubart, M., and Zipes, D. P. (2005). Mechanisms of sudden cardiac death. J. Clin. Invest. 115, 2305-2315. doi: 10.1172/JCI26381

Siscovick, D. S., Raghunathan, T. E., King, I., Weinmann, S., Wicklund, K. G., Albright, J., et al. (1995). Dietary intake and cell membrane levels of long-chain n-3 polyunsaturated fatty acids and the risk of primary cardiac arrest. JAMA 17, 1363-1367. doi: 10.1001/jama.1995.035301700 43030

Smith, P. J., Blumenthal, J. A., Babyak, M. A., Georgiades, A., Sherwood, A., Sketch, M. H. Jr., et al. (2009). Association between n-3 fatty acid consumption and ventricular ectopy after myocardial infarction. Am. J. Clin. Nutr. 5, 1315-1320. doi: 10.3945/ajcn.2008.26829

Sridhar, A., Nishijima, Y., Terentyev, D., Terentyeva, R., Uelmen, R., Kukielka, M., et al. (2008). Repolarization abnormalities and afterdepolarizations in a canine model of sudden cardiac death. Am. J. Physiol. Regul. Integr. Comp. Physiol. 295, R1463-R1472. doi: 10.1152/ajpregu.90583.2008

Varkevisser, R., Wijers, S. C., Van Der Heyden, M. A., Beekman, J. D., Meine, M., and Vos, M. A. (2012). Beat-to-beat variability of repolarization as a new biomarker for proarrhythmia in vivo. Heart Rhythm. 10, 1718-1726. doi: 10.1016/j.hrthm.2012.05.016

Verkerk, A. O., van Ginneken, A. C., Berecki, G., den Ruijter, H. M., Schumacher, C. A., Veldkamp, M. W., et al. (2006). Incorporated sarcolemmal fish oil fatty acids shorten pig ventricular action potentials. Cardiovasc. Res. 3, 509-520. doi: 10.1016/j.cardiores.2006.02.022

Wagner, S., Maier, L. S., and Bers, D. M. (2015). Role of sodium and calcium dysregulation in tachyarrhythmias in sudden cardiac death. Circ. Res. 12, 1956-1970. doi: 10.1161/CIRCRESAHA.116.304678

Wang, D., and O'Horo, J. (2011). Antifibrotic effects of omega-3 fatty acids in the heart: one possible treatment for diastolic heart failure. Trends Cardiovasc. Med. 3, 90-95. doi: 10.1016/j.tcm.2012.03.005

Wilhelm, M., Tobias, R., Asskali, F., Kraehner, R., Kuly, S., Klinghammer, L., et al. (2008). Red blood cell omega-3 fatty acids and the risk of ventricular arrhythmias in patients with heart failure. Am. Heart J. 6, 971-977. doi: 10.1016/j.ahj.2007.11.045

Xiao, Y. F., Kang, J. X., Morgan, J. P., and Leaf, A. (1995). Blocking effects of polyunsaturated fatty acids on $\mathrm{Na}^{+}$channels of neonatal rat ventricular myocytes. Proc. Natl. Acad. Sci. U.S.A. 24, 11000-11004.

Conflict of Interest Statement: The authors declare that the research was conducted in the absence of any commercial or financial relationships that could be construed as a potential conflict of interest.

Copyright (๑) 2016 Bonilla, Nishijima, Vargas-Pinto, Baine, Sridhar, Li, Billman and Carnes. This is an open-access article distributed under the terms of the Creative Commons Attribution License (CC BY). The use, distribution or reproduction in other forums is permitted, provided the original author(s) or licensor are credited and that the original publication in this journal is cited, in accordance with accepted academic practice. No use, distribution or reproduction is permitted which does not comply with these terms. 